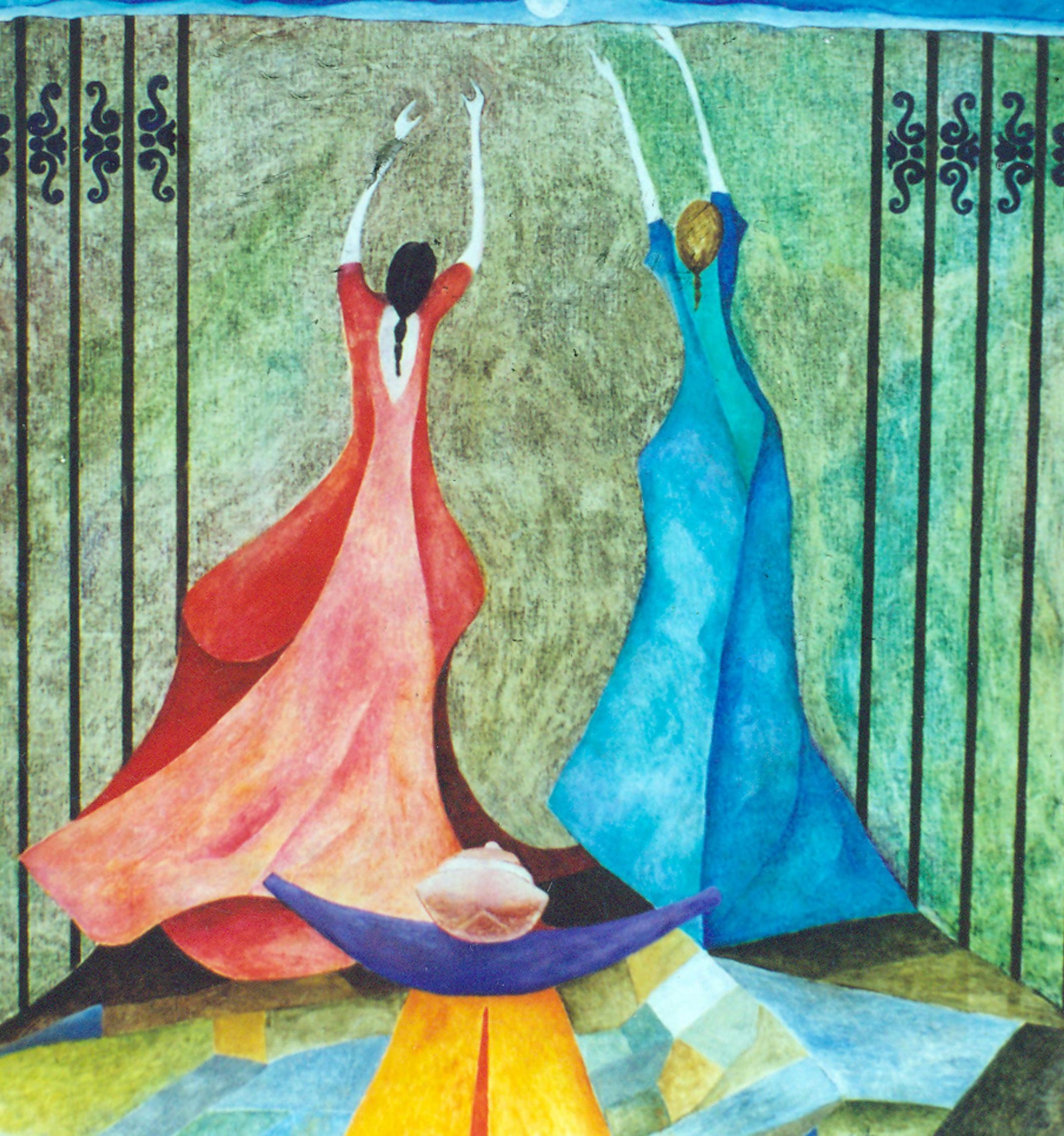




\section{Fragmentación y segregación urbana en el Distrito Central, Honduras}

Marysabel Zelaya Ochoa*

RESUMEN. Hasta la década de los setenta la fragmentación urbana en el Distrito Central no era tan evidente, la Plaza Central era considerada el centro; constituía el punto de origen apartir del cual de daba identidad y orientación a la ciudad. Era la zona de mayor jerarquía urbana, sede del poder político, económico, religioso y social. Sin embargo, con los cambios introducidos a partir de los ochenta la situación cambia, la ciudad deja de representar un espacio contínuo para dar lugar a una ciudad caracterizada por la fragmentación y segregación urbana.

Palabras claves: segregación urbana, fragmentación, barrio cerrado, espacio público, exclusión social.

ABSTRACT. In the Central District until the seventies decade the urban fragmentation was not so evident, Central Park was considered the center, constituted the point of origin and identity and orientation given to the city, was the area of greatest urban hierarchy, home of political, economic, religious and social. However, with the changes made since the eighties, the situation changes, the city cease to represents a continuous space to make way for a city characterized by fragmentation and urban segregation.

Keywords: urban segregation, fragmentation, gated community, public space, and social exclusion.

\section{Introducción}

Durante los últimos treinta años se observa como el proceso de urbanización que se desarrolla en el Distrito Central reviste características particulares que marcan un proceso de fragmentación y segregación urbana, el cual se manifiesta en la constitución de nuevas centralidades que giran en torno a la prestación de servicios comerciales y financieros y al surgimiento de barrios cerrados como nueva forma de segregación residencial. El creciente surgimiento de este tipo de urbanizaciones está ligado a diversas causas entre las que sobresalen la violencia e inseguridad que se vive en la capital y el deseo de una parte de la población de alcanzar un estatus social y un estilo de vida diferente al del resto de la población.

Uno de los elementos que incide en el proceso de fragmentación y segregación urbana es el factor socioeconómico producto de ello se establecen notables diferencias entre las viviendas, acceso a servicios e infraes- tructura básica y a los espacios públicos en los diferentes barrios y colonias. En este proceso la ciudad pierde su carácter abierto, heterogéneo y accesible para convertirse en una ciudad cerrada, amurallada, trancada e inaccesible en donde predomina la polarización social caracterizada por una demarcación física y simbólica del territorio que genera diversas expresiones de violencia y un progresivo deterioro de los espacios de convivencia social.

El objetivo general de este artículo consiste en explicar el proceso de fragmentación y segregación urbana experimentado en el Distrito Central y su relación con los procesos de exclusión social. El artículo surge en el contexto de la investigación de tesis doctoral "La Crisis de los Espacios Públicos Urbanos en el Distrito Central, que fue apoyado como parte de un convenio de cooperación entre la Universidad Nacional Autónoma de Honduras y el National Centre of Competence in Research (NCCR), North South, JACS-CCA iniciativa que para Centroamérica, México y El Caribe centró su interés en 
la discusión de temas relacionados con una línea temática denominada formas de vida y territorio: exclusión social, fronteras internas, segregación, fragmentación e inseguridad.

\section{Metodología}

El articulo parte de un enfoque descriptivo explicativo, se hizo uso de fuentes de información secundaria a nivel de textos y revistas científicas para identificar los enfoques teóricos conceptuales que permiten comprender diferentes ópticas bajo las cuales se explica el fenómeno social, además se desarrolló trabajo de campo que permitió identificar las formas de segregación y fragmentación urbana que ha sufrido el Distrito Central.

\section{Enfoques teóricos}

\subsection{La fragmentación y segregación urbana}

La fragmentación es un proceso inherente a la conformación de la ciudad, esta se va construyendo como un mosaico en donde el suelo tiene diferentes usos y formas. En ese sentido, se distingue en el espacio del Distrito Central áreas residenciales, comerciales, industriales y recreativas, entre otras.

Por una parte la fragmentación urbana se asocia a una ruptura, separación o distanciamiento social en la ciudad, estudiado básicamente a través de la idea de segregación. Esta aproximación considera que la actual dinámica urbana, desde el comportamiento del mercado de suelos, las iniciativas inmobiliarias, el surgimiento de estilos de vida, hasta las transformaciones en el mercado de trabajo, la polarización social, el aumento de la violencia y la inseguridad, lleva a una separación social en el espacio que se refleja en el surgimiento de barrios cerrados o similares (Link, F, 2008, pág 29, en Revista Ciudades nº 77).

En general la ciudad va creando barreras cada vez más rígidas que muestran una fuerte separación entre quienes pueden adaptarse al nuevo contexto y quienes simplemente se quedan fuera de él.
Estela Valdés (2007) en su ponencia Fragmentación y Segregación Urbana identifica dos líneas de análisis: por un lado, aquella que se halla ligada a procesos de desigualdad social y barreras materiales y/o inmateriales; por el otro, la que se relaciona con las discontinuidades en el proceso de expansión urbano respecto de la trama, producto de los procesos de metropolización.

La primera línea de análisis reconoce autores como David Harvey, Prévót-Shapira y Vidal Rojas, entre otros. Para Harvey (1997), las ciudades en la actualidad han dejado de planificarse en su conjunto para sólo abocarse a diseñar partes de ellas como resultado de la especulación inmobiliaria y sin ningún tipo de previsión; advierte que, por un lado aparece la miseria y la corrupción; y por el otro, lugares hermosos de diseños arquitectónicos realizados por especialistas famosos pero cuyos habitantes no tienen idea sobre lo que sucede en los sectores más pobres de la ciudad (Harvey, 1997, pág 12).

Prévot-Shapira (2000) considera que algunas ciudades latinoamericanas son representativas de un modelo de ciudad más disperso y menos jerárquico, que sustituye a la ciudad orgánica, esto es, la ciudad fragmentada y que involucra los siguientes componentes: a) espaciales, como la desconexión física y discontinuidades morfológicas; $b$ ) dimensiones sociales, como el repliegue comunitario y lógicas exclusivas; y c) políticas, tales como la dispersión de actores y autonomización de dispositivos de gestión y regulación urbana. De esta manera, la ciudad orgánica "ha estallado en múltiples unidades y ya no existe la unificación del conjunto" (Prévot-Shapira, 2000, pág 34-35).

Para Vidal Rojas la fragmentación urbana es un proceso territorial que se construye a través de tres subprocesos: fragmentación social, fragmentación física y fragmentación simbólica y en cualquiera de los casos supone la independencia de las partes (fragmentos) en relación al todo (sistema urbano). La fragmentación física -entendida en términos físico -relacionales-puede ser definida "como la tendencia de la estructura de la ciudad hacia una pérdida de la coherencia y de cohesión del todo a 
causa de una disociación de las partes que la componen" (Vidal, R. 1997, pág 5).

En la otra línea de análisis, se ubican autores como Borsdorf (2003), De Mattos (2001), y Ciccollella (2002). Según la mirada de Borsdorf (2003) de la ciudad polarizada, propia de los años de industrialización sustitutiva en América Latina, se pasa a la ciudad fragmentada de la actualidad. En el primer caso, la configuración de la ciudad hasta principio de los setenta mostró procesos espaciales ligados a las líneas ferroviarias y autopistas que reforzaron el crecimiento de algunos sectores; los grupos de mayores recursos se desplazaron hacia la periferia y aparecieron en algunas de las ciudades latinoamericanas, ya al final del período, los primeros centros comerciales -Shopping centres-y barrios de lujo (countries) con estilos importados de las ciudades estadounidenses. Sin embargo, en este período, la estructuración del espacio urbano responde todavía a la industrialización y a la presencia del Estado intervencionista en cuestiones relativas a la planificación, como así también a la fuerte migración rural (Borsdorf, 2003, pág 48).

De Mattos (2001), comparte esta visión - al igual que otros autores - y afirma que las ciudades se constituyen como estructuras suburbanizadas y policéntricas con una tendencia a la "angelinización" como el ejemplo paradigmático (De Mattos, 2001, pág 3).

Ciccollella considera que el modelo de la ciudad actual ha dejado de lado la ciudad compacta para dar lugar a otra más fragmentada, de crecimiento celular y a la que se denomina metrópolis expandida, postsocial, metápolis o ciudad difusa, tal alguno de los nombres con los que lo han denominado distintos autores (Ciccollella, P 2002, pág 204).

Consecuencia directa de la fragmentación urbana es la segregación urbana. Castells (1999) la define como la tendencia a la organización del espacio en zonas de fuerte homogeneidad social interna y de fuerte disparidad social entre ellas, entendiéndose esta disparidad no sólo en términos de diferencia, sino de jerarquía. En este sentido, la estratificación social origina también estratificación espacial que se traduce en áreas urbanas segregadas y ocupadas por grupos sociales semejantes viviendo en entornos morfológicos también semejantes (Estébanez, 1992, pág 574).

En términos sociológicos, segregación significa la ausencia de interacción entre grupos sociales. En un sentido geográfico, significa desigualdad en la distribución de los grupos sociales en el espacio físico (Rodríguez Vignoli, 2001, pág 11).

La manifestación tangible del fenómeno de segregación urbana se presenta en la segregación espacial residencial, entendida como el distanciamiento o separación de grupos poblacionales en el espacio urbano, este fenómeno se evidencia en la aglomeración geográfica de familias con características similares generalmente relacionadas con la condición étnica, origen migratorio o condición socioeconómica.

En el caso del Distrito Central las desigualdades sociales de ingreso determina en gran medida la segregación residencial.

La segregación residencial parece inherente a la vida urbana, pero en la actualidad pareciera tener mayor visibilidad. La principal razón por la cual la segregación residencial está en el tapete es por las adversidades que se le imputan cuando su raíz es socioeconómica, es decir, segregación residencial socioeconómica (SRS). A grandes rasgos, la SRS actúa como mecanismo de reproducción de las desigualdades socioeconómicas, de las cuales ella misma es una manifestación (Kaztman, 2001; Dureau et al., 2002).

En términos estrictamente socio-económicos, los mecanismos de desigualdad social y desarrollo geográfico desigual va inferiorizando a personas, grupos y comunidades en integración e interdependencia con sus contextos vitales (Álvarez, H 2005, pág 5).

La segregación residencial de tipo socio-económica es considerada para numerosos autores como una de las 
dimensiones empíricas y teóricas más importantes de la exclusión social, en donde la pobreza confronta las sociedades e implica la división de la sociedad como resultado de las desigualdades sociales existentes. Esta situación está produciendo la reducción de los espacios de interacción entre los grupos socioeconómicos deteriorando la vida en comunidad a tal grado que priman acciones de violencia, inseguridad, temor y desconfianza hacia todos los que son diferentes en su condición social.

La Escuela de Sociología Urbana de Chicago una de las primeras en abordar el estudio de la temática; la definió como una concentración de tipos de población dentro de un territorio dado y se aplicó al estudio de la distribución espacial de minorías étnicas en grandes ciudades de los EE.UU. (Mackenzie, 1925).

La fragmentación urbana resulta del proceso de expansión, se va creando una ciudad dispersa que genera diferenciación del territorio y en muchas ocasiones desconexión entre los fragmentos. A la par de este proceso se genera la segregación urbana que implica la unión y diferenciación cultural y socioeconómica, basado en las fuerzas de producción y en la división social del trabajo que territorialmente son evidentes a través de características como el uso del suelo, los servicios, equipamiento y la infraestructura.

Todo el proceso de segregación urbana genera asimismo una auto segregación que quizá en un primer momento este marcada por barreras físicas, tangibles que impiden el acceso a ciertos espacios y bienes, pero que con el tiempo también se marca por barreras mentales, intangibles que acentúan la desigualdad social y la exclusión.

\section{El Distrito Central y las nuevas formas de fragmentación y segregación urbana}

El Distrito Central, espacio geográfico en el que se ubica la capital de la república constituida por las ciudades de Tegucigalpa y Comayagüela atraviesa por crecientes transformaciones físicas, sociales y culturales que muestran una capital que está cambiando sin tener el norte, ni la brújula que dirija su camino, esto genera una problemática urbana que no ha sido abordada con la seriedad que merece.

Originalmente, Tegucigalpa fue construida bajo los parámetros de la arquitectura colonial española que se desarrolló centralizando las funciones de la ciudad como organismo administrativo básico de la colonia. Dicha construcción se basó en las ordenanzas de Felipe II que contemplaba dentro del trazado de la ciudad la construcción de la Plaza Mayor, la Iglesia y el Cabildo alrededor de los cuales se levanta el resto de la ciudad.

Los cambios experimentados por las ciudades coloniales son evidentes tanto en su morfología como en su funcionamiento institucional y en las relaciones sociales que se dan en su interior. A nivel de estructura se presentan cambios de uso del suelo que se manifiestan en la creciente transformación de la ciudad, de igual manera estos cambios físicos van acompañados de fenómenos sociales como la segregación espacial y residencial.

Quizá hasta la década de los setenta la fragmentación urbana en el Distrito Central no era tan evidente, a partir de los ochenta se observan algunos cambios, para el caso inicia la construcción de bulevares, proyectos habitacionales en la periferia de la ciudad y los primeros centros comerciales que se presentan como un fragmento con sentido en el nuevo espacio urbano, alrededor de ellos poco a poco se van generando nuevas centralidades. Hacia los noventa se gesta la construcción de las sedes de los poderes del Estado fuera del centro histórico.

Entre los ochenta y noventa se incrementa el crecimiento de cinturones de miseria dando lugar al surgimiento de colonias urbano marginales producto de procesos de recuperación de tierras. Se consolidará entonces la estructura de un modelo urbano descompensado por sus fuertes desequilibrios rotacionales especialmente en lo relacionado al acceso a servicios públicos y la consolidación de un modelo de ciudad desarticulado territorialmente con una fuerte fragmentación urbana en donde se fortalece la segregación urbana residencial.

Hacia la primera década del siglo XXI el proceso de fragmentación urbana se ha consolidado por medio del 
surgimiento de urbanizaciones cerradas en distintas áreas de la capital que establecen un fuerte distanciamiento tangible e intangible con el resto de barrios y colonias capitalinas, estas nuevas urbanizaciones se presentan como soluciones individuales para aquellas familias que se encierran en sectores seguros de la capital en donde lo público ha sido desplazado por lo privado y lo colectivo por lo individual.

Fortaleciendo este proceso, en el Distrito Central está en boga la construcción de centros comerciales de inversión privada, estos presentan un mundo irreal en comparación a la realidad socioeconómica de la ciudad, se desarrollan como un fragmento, como nuevos centros de la ciudad que se valen por sí mismo y que está desconectado del resto y de su realidad.

\subsection{Las nuevas centralidades en el Distrito Central}

En la medida en que Tegucigalpa y Comayagüela ha crecido, se desarrollan procesos simultáneos que le dieron un nuevo aspecto a su trama urbana. Uno de estos procesos es el surgimiento de una policentralidad constituyendo un cambio notable sobre todo porque hace sólo 35 años todo giraba alrededor del centro fundacional.

La categoría centralidad espacial hace referencia a la jerarquización o priorización que se realiza de un lugar específico. El lugar central de la ciudad se ha caracterizado por ser el espacio donde se encuentran las máximas expresiones sociales, económicas y culturales de la población.

La policentralidad es entendía como un significativo aumento del número de funciones y actividades que habían estado localizadas en los centros tradicionales y que ahora se desplazan hacia nuevos lugares del territorio metropolitano (De Mattos, 2002, pag 26).

Esta categoría de alguna manera es mediada por factores como acceso, distancia, pero también se tiene en cuenta las percepciones asociadas a las escalas de preferencia de la gente.

En el caso de Tegucigalpa y Comayagüela se parte de una centralidad fundacional, que dio lugar a la acumula- ción de servicios en las cercanías tales como: energía eléctrica, calles, avenidas, saneamiento, intercambio comercial y financiero, servicios educativos y de salud, entre otras.

Con el tiempo se ha desarrollado una centralidad funcional marcada por la necesidad de integración en el marco de una ciudad segregada, dando lugar a nuevas centralidades que se ubican en lugares estratégicos de la capital generalmente construido desde el mundo de lo privado y teniendo como punto de partida los grandes centros comerciales.

Un recorrido por las ciudades de Tegucigalpa y Comayagüela, permitió identificar las principales instituciones y servicios establecidos en distintas áreas de la capital y elaborar una representación gráfica. Básicamente se identifican 6 centralidades; se parte del Centro Histórico como centralidad fundacional, la zona del bulevar Morazán, la zona donde se ubica Casa Presidencial y Bulevar Suyapa, la zona de la Kennedy y Bulevar Fuerzas Armadas, la zona del Aeropuerto Toncontín y la zona del mercado Belén (Mapa 1).

El proceso de crecimiento urbano del Distrito Central experimentado bajo los efectos de la globalización afianza el desarrollo de nuevas centralidades urbanas contribuyendo de manera directa con la acentuación de los procesos de fragmentación social y urbana lo que paulatinamente le niega a la población la posibilidad de encuentro y de intercambio generando una ruptura, separación o distanciamiento social en la ciudad que incide negativamente en la resolución de la problemática social que experimenta.

\subsection{Segregación residencial y socioespacial en el Distrito Central}

En el Distrito Central la segregación residencial y espacial no se ha desarrollado siguiendo un patrón territorial, en otras palabras, esta se presenta de manera diseminada en la ciudad. Se puede encontrar colonias dirigidas a grupos poblacionales de alto poder adquisitivo a la par o muy cerca de barrios populares o colonias urbano marginales; esta situación da lugar a zonas homogéneas en un contexto heterogéneo en donde la interacción 
Mapa 1. Distrito Central: nuevas centralidades urbanas

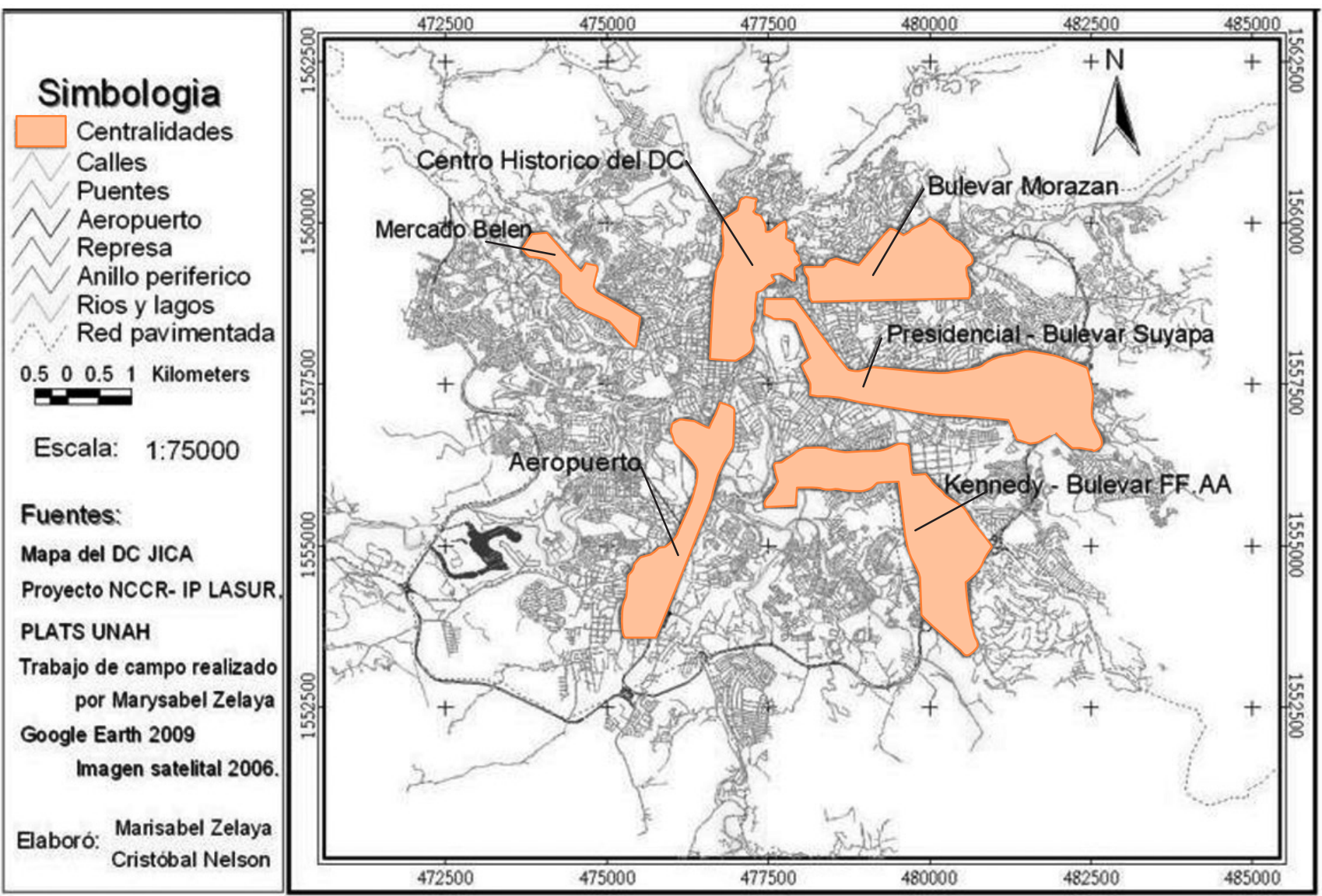

Fuente: Elaboración propia, trabajo de campo, Proyecto NCCR, LASUR PLATS UNAH.

entre los grupos sociales es muy limitada y en algunos casos casi nula.

Evidencia de lo anterior es el caso del municipio del Distrito Central cuya característica fundamental ha sido el crecimiento desordenado, con un ordenamiento territorial casi ausente, aunque desde los años setenta se da el primer esfuerzo organizado institucionalmente para orientar la expansión y desarrollo urbano del Distrito Central a través de METROPLAN ${ }^{1}$.

A partir de la década de los sesenta el Distrito Central experimenta un notable crecimiento poblacional urbano, se pasó de tener una población de 99,948 a 164,941, representando un crecimiento poblacional del 65\%, es a partir de este periodo que se van generando cambios en la estructura de la ciudad principalmente en la distribución

1. METROPLAN: Plan de Desarrollo Metropolitano del Distrito Central del uso del suelo en donde el uso residencial presenta un gran peso (Cuadro 1).

Durante el gobierno del Dr. Ramón Villeda Morales (1957-1963) se implementan políticas estatales de construcción de vivienda de interés social por medio del Instituto Nacional de la Vivienda (INVA), esta institución desarrolló proyectos habitacionales dirigidos a familias de obreros, los proyectos se ubicaron en zonas distantes del centro de la ciudad, hecho de una u otra manera dio lugar a la desconcentración del Centro Histórico iniciando el crecimiento de la ciudad hacia la periferia (Cuadro 2).

Los espacios urbanos ubicados entre el Centro Histórico y las primeras colonias de interés social: Col. 21 de Octubre y Col. Kennedy poco a poco fueron ocupados por otros proyectos habitacionales. Para el caso entre la colonia 21 de Octubre y el Centro Histórico se 
Cuadro 1. Distrito Central: población total por censo y tasas de crecimiento intercensal

\begin{tabular}{|c|c|c|c|}
\hline Censo & Población & \multicolumn{2}{|c|}{ Tasa crecimiento intercensal } \\
\hline 1950 & 99,948 & & \\
\hline 1961 & 164,941 & $1950-1961$ & 4.6 \\
\hline 1974 & 305,387 & $1961-1974$ & 4.8 \\
\hline 1988 & 585,686 & $1974-1988$ & 4.6 \\
\hline 2001 & 850,227 & $1988-2001$ & 2.08 \\
\hline 2013 & & $2001-2013$ & \\
\hline
\end{tabular}

Fuente. Manuel Flores Fonseca, 50 años de crecimiento demográfico Hondureño, 2003.

Cuadro 2. Distrito Central: colonias construidas por el INVA

\begin{tabular}{|l|c|c|}
\hline Colonia & Año de inicio & No de viviendas \\
\hline 21 de Octubre & 1957 & 589 \\
\hline John F. Kennedy & 1964 & 3,638 \\
\hline Las Brisas & 1966 & 152 \\
\hline San José del Pedregal & 1968 & 902 \\
\hline La Laguna & 1962 & 170 \\
\hline 15 de Septiembre & 1977 & 97 \\
\hline San José de la Peña & 1977 & 584 \\
\hline San José de la Vega & 1978 & 611 \\
\hline Faldas de El Pedregal & 1979 & 258 \\
\hline Hato de En medio & 1980 & 2,108 \\
\hline El Sitio & 1982 & 972 \\
\hline
\end{tabular}

Fuente: Elsa Lily Caballero Zeitún, El Metroplan y la Política de Vivienda 1976-1984

construyeron colonias como la San Carlos, Las Minitas, Col Lara, entre las más relevantes, en estos vecindarios residían grupos sociales de alto poder adquisitivo por lo que esta zona llego a ser considerada como área selectas de la capital. En el caso de los espacios ubicados entre la Col. Kennedy y el Centro Histórico se construyeron las colonias Miraflores, El Hogar, Las Colinas, Miramontes todas financiadas con capital privado o por cooperativas de vivienda como FECOHVIL.

Hasta los años setenta los patrones de segregación residencial y espacial no tenían la magnitud que manifiestan hoy en día, la ciudad se concentraba prácticamente alrededor del centro histórico, la plaza central y espacios adyacentes. La gente de diferentes grupos sociales compartía eventos culturales, políticos, sociales y la vida cotidiana en los mismos espacios públicos. En tal sentido, aunque la diferenciación social fuese evidente no lo era la segregación residencial y espacial.

Durante los ochenta con los cambios experimentados por la ciudad y más aún en los noventa la población se desplaza a sectores periféricos de la ciudad, algunos urbanizados, otros en condiciones de precariedad urbana.

Es a finales de los noventa e inicios del siglo XXI que un porcentaje de la población capitalina decide establecer su residencia en los municipios cercanos al Distrito Central (Valle de Ángeles, Santa Lucía, Santa Ana, Ojojona) en donde puede gozar de la tranquilidad y las ventajas climáticas de la zona, pero de igual manera del acceso a servicios públicos de aceptable calidad. El valor del suelo en estas zonas ha aumentado dando lugar incluso a desarrollo de numerosas urbanizaciones cerradas.

En el caso del Distrito Central la segregación residencial y socioespacial está fuertemente relacionada con los ingresos económicos, de ahí que se mencione el fenómeno de segregación residencial socioeconómica, en donde se distinguen la tendencia de un grupo a concentrarse en algunas áreas y la conformación de áreas socialmente homogéneas.

\subsection{Surgimiento de urbanizaciones cerradas en} el Distrito Central

Actualmente la empresa privada promueve la construcción de urbanizaciones cerradas como nuevo elemento dentro de la estructura urbana y un elemento transformador del espacio residencial que constituye expresión clara de exclusión residencial urbana. El surgimiento de urbanizaciones cerradas en la capital es un fenómeno relativamente reciente, sin embargo, los últimos años registran un importante aumento, este escenario está relacionado en alguna medida por la desregulación del mercado del suelo.

Esta situación coincide con el esquema planteado por Harvey donde la globalización se asocia directamente con el dominio económico neoliberal e involucra una creciente desregulación de la economía y la actividad del sector público, factores que a nivel urbano se manifiestan en la liberalización del mercado del suelo y la especulación inmobiliaria (Borsdorf, Hidalgo, 2004, pag 28). 
El incremento de urbanizaciones cerradas es un fenómeno urbano de gran crecimiento en el continente americano, se apoya en la fragmentación del territorio y en la especialización del uso del suelo pero también presenta una tendencia hacia el control de las relaciones en el espacio físico de las ciudades, presentándose como una nueva forma de privatización del espacio urbano. En el caso del DC han surgido urbanizaciones cerradas cuyos muros perimetrales crean fragmentos dentro de la ciudad, en algunos casos pequeñas ciudadelas que aíslan a la población, este fenómeno afecta considerablemente el uso de los espacios públicos y se va perdiendo el acceso a la libre circulación dentro de la ciudad.

El origen de las urbanizaciones cerradas en el Distrito Central está ligado a diversas causas, entre las cuales se identifica en primera instancia la violencia e inseguridad
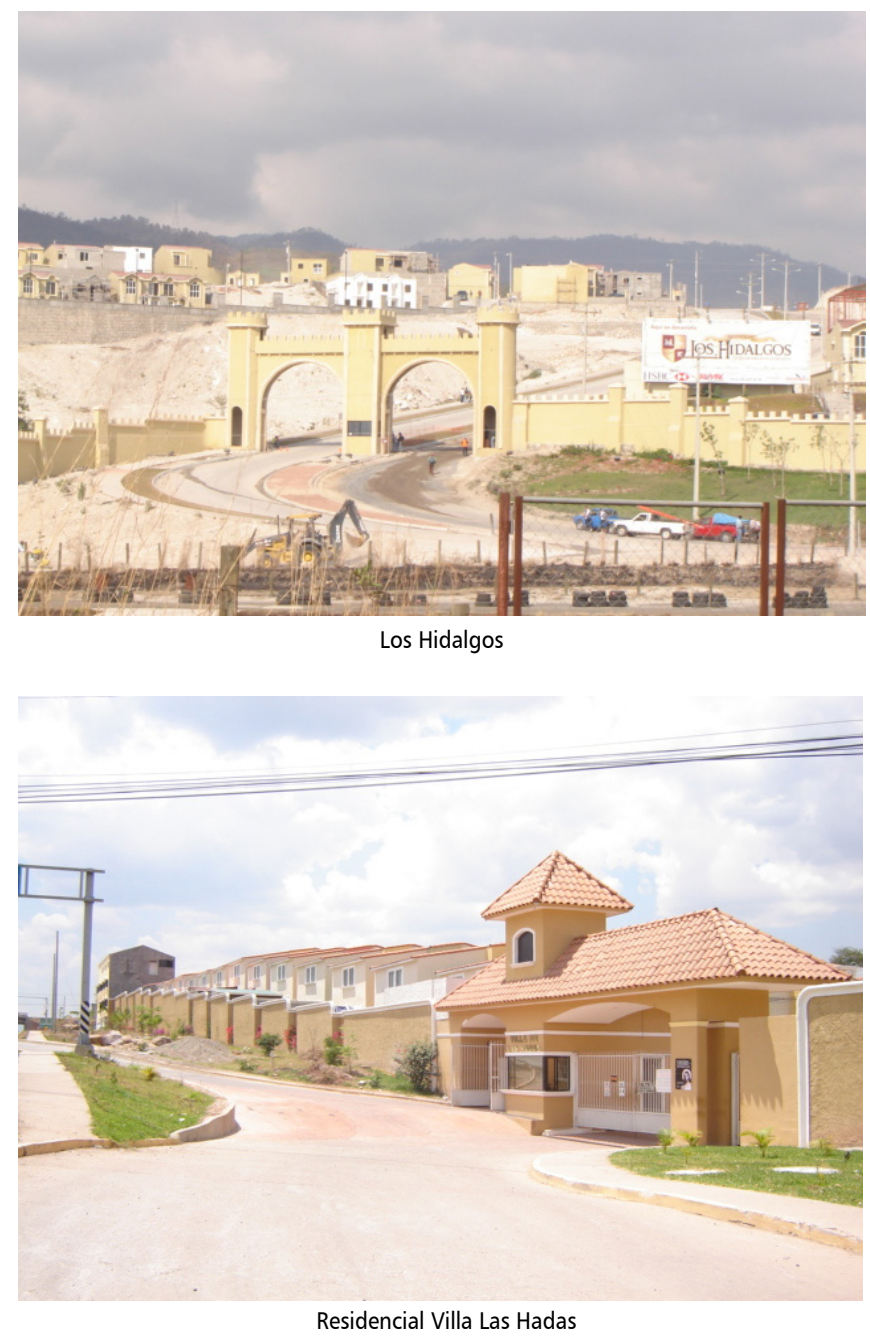

sobre todo porque en la última década se muestra un incremento en las estadísticas de criminalidad con serias repercusiones sobre la vida y la seguridad de los bienes de los ciudadanos.

Según Caldeira, desde mediados de los años ochenta, el progreso de la criminalidad y del miedo ha servido para legitimar el modelo de segregación residencial que representa el barrio cerrado (Caldeira, 1996, pag 65).

En aquellas urbanizaciones dirigidas a estratos medios-altos y altos se desarrollan las urbanizaciones de circuito cerrado caracterizados por estar rodeados o cercados por muros perimetrales contando con mecanismos de control las 24 horas, servicio que todos los y las vecinas pagan, generalmente estas urbanizaciones se ubican en la periferia de la ciudad adyacentes a las vías de acceso y en zonas de alta renta.

Los nuevos emprendimientos urbanos en el Distrito Central se promocionan haciendo énfasis en la provisión de seguridad a sus residentes. En la actualidad las palabras cerrados, seguridad y vigilancia forman parte de la promoción de los nuevos conjuntos de viviendas que se construyen en la capital. Otro factor considerado en el mercado está relacionado con el deseo de la población de alcanzar un estatus social y un nivel de vida diferente y hasta exclusivo que le asignan a la población de estos conjuntos residenciales un carácter de distinción.

Según Angela Giglia, la privatización del espacio público no es únicamente una forma de protegerse frente a la inseguridad creciente, sino también una manera de diferenciarse del entorno para "distinguirse", y mantener en el interior del espacio cerrado un modo de vida con características especifica (Giglia, A, 2002, pag 24).

Otro aspecto a considerar en las urbanizaciones planificadas desde su origen como cerradas está relacionado con el tamaño. En el Distrito Central se observan urbanizaciones muy pequeñas como Quinta San Ignacio con 8 viviendas, hasta megaproyectos como Los Hidalgos, urbanización diseñada para construir más de 2000 viviendas distribuidas en 10 circuitos. Esta urbanización 
en inicio se concibió bajo el concepto de una pequeña ciudad, como un lugar del que sólo se necesita salir, para trasladarse a su trabajo, puesto que en su diseño se piensa contar con supermercado, centro comercial, iglesia, clubes privados, centros escolares y áreas verdes. Además la muralla que rodea la urbanización es típica de las antiguas ciudades medievales. Esta urbanización contempla viviendas dirigidas a distintos grupos e intereses. Se presentan desde apartamentos para solteros y parejas, viviendas de un solo nivel, dos niveles, hasta viviendas exclusivas con acceso a clubes privados, esto hace pensar en el nivel de exclusión que se verá relejado aun entre los mismos habitantes de la urbanización.

Según Judd (1995) algunos estudios comparan las urbanizaciones cerradas con las ciudades amuralladas del mundo medieval, en donde las murallas se contraían para mantener a raya a las hordas (Judd, 1995, pag 155, 160).

Más allá de aquellas urbanizaciones que desde su origen fueron concebidas como barrios cerrados, en el Distrito Central, los barrios y colonias capitalinas de todos los estratos sociales han ido cerrando sus vecindarios al libre tránsito de la población por medio del establecimiento de controles de seguridad, unos más efectivos que otros. Esta situación ha generado conflictos entre la ciudadanía a tal grado que la Asociación Metropolitana del Distrito Central por medio de la Unidad de Movilidad Urbana maneja el Proyecto Barrio Seguro y ha establecido un reglamento que regula la emisión de permisos y las condiciones bajo las cuales operaran este tipo de urbanizaciones, sin embargo, pese ello muchos barrios y colonias del DC han cerrado su acceso y operan en condiciones de ilegalidad.

En este contexto se presenta en el Mapa 2 la ubicación de los barrios y colonias capitalinas que actualmente cuentan con el permiso autorizado por la Unidad de Movilidad Urbana de la AMDC.

Cabe señalar que el 29\% de los barrios y colonias de Distrito Central se manejan como barrios cerrados y la tendencia que se proyecta esta dirigida al crecimiento de esta modalidad.
La segregación residencial y socioespacial en el Distrito Central se traducen en cambios ostensibles en la organización y las formas de producción de espacio urbano que se manifiestan en la proliferación de grandes proyectos inmobiliarios conducidos por el capital privado en donde la característica primordial es la privatización del espacio urbano, que pasan a ser controlados por dispositivos de seguridad regulando y controlando el libre acceso, a la larga se genera una crisis de integración, se limita la solidaridad y como lo menciona Davis (2003) se va produciendo un apartheid urbano evidenciado en el hecho de que sólo determinados grupos sociales tienen acceso a los espacios más exclusivos de la ciudad, generando una creciente desigualdad social y consecuentemente exclusión social, en donde los más pobres son los más postergados frente a los procesos de desarrollo local y municipal.

Las urbanizaciones cerradas buscan fomentar una sensación de comunidad, sin embargo, este hecho no funciona por inercia entre los residentes de estas urbanizaciones, en ocasiones el sentido de comunidad sólo está ligado al hecho de que pertenecen al mismo mercado inmobiliario. En otras palabras el pertenecer a la misma urbanización no implica asegurarse vínculos de buena vecindad entre que los que ahí viven.

El fenómeno de la segregación espacial y residencial manifestado en el surgimiento de urbanizaciones cerradas ha redefinido la configuración de las ciudades de Tegucigalpa y Comayagüela una de estas consecuencias es evidente en la privatización de espacios en la ciudad y la pérdida de los espacios públicos. Las calles y avenidas que antes eran de acceso público en el Distrito Central en la actualidad está controlada en la mayor parte de los casos con plumas y portones metálicos o trancas que limitan el libre acceso de la población a estos espacios sobre todo en las urbanizaciones de clase media y media alta.

Se ha considerado que las urbanizaciones cerradas violan el derecho constitucional a la libre circulación, al impedir la libre circulación (CYTED, 2002:482). 
Mapa 2. Barrios y colonias del Distrito Central legalmente cerrados autorizados por la Gerencia de Movilidad Urbana y Transporte AMDC 2015.

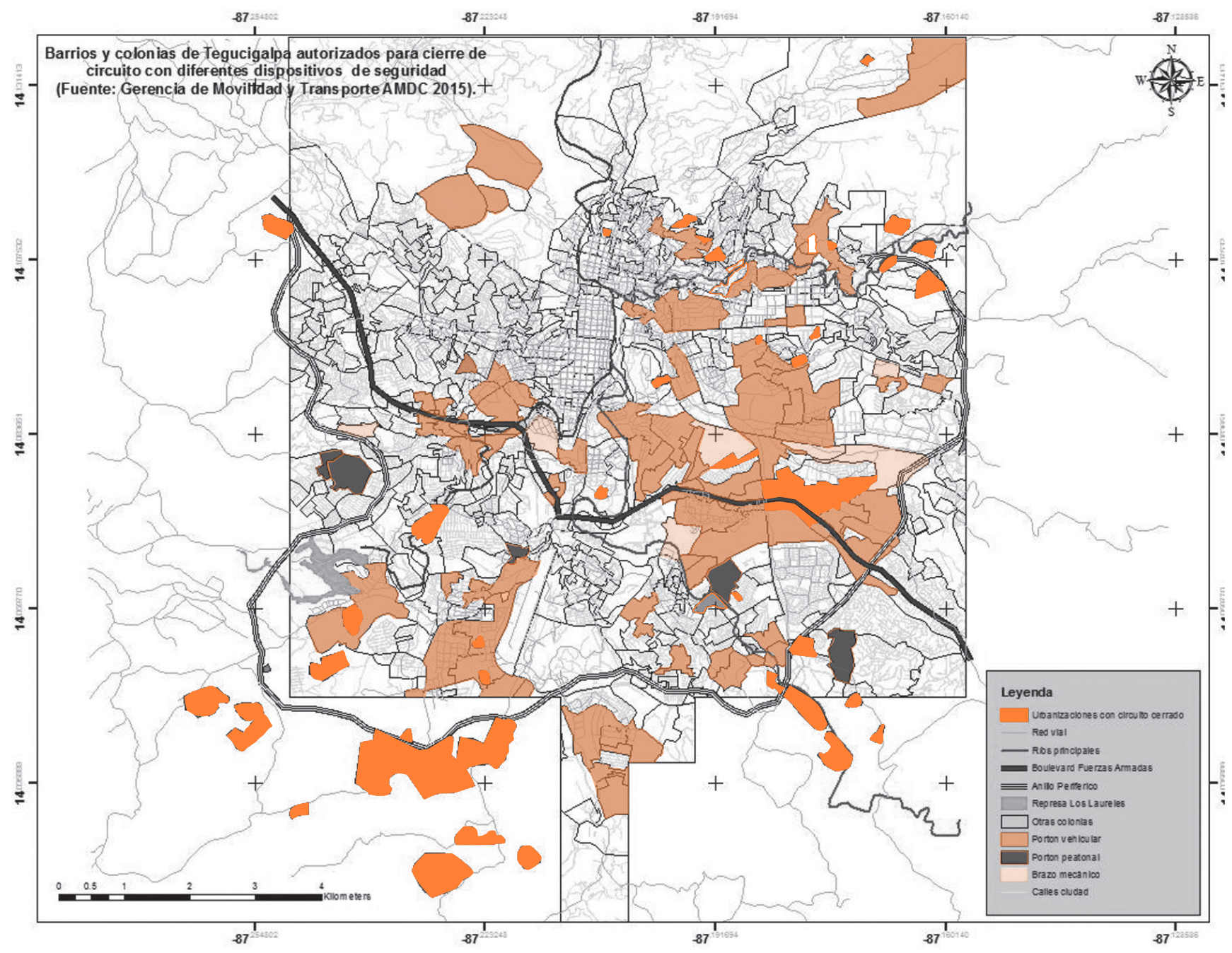

Estas formas de segregación ligadas a la distribución de las clases sociales en el territorio ha generado consecuencias en términos del tejido social y del proceso de segregación social urbana implícito en su desarrollo. Las urbanizaciones cerradas denotan un declive del espacio público urbano, de alguna manera el fraccionamiento y la desintegración de la ciudad.

Desde la óptica externa la exclusión se hace más evidente, ya el muro o la barrera por si misma establece un símbolo de limite, separación y hasta aislamiento que refleja la diferencia de oportunidades entre la población de la ciudad, disminuyendo la posibilidad de interacción entre los diversos grupos sociales.
En el contexto de las urbanizaciones cerradas se crea una doble óptica de exclusión que se refleja en la desconfianza hacia el otro. Los de adentro tienden a aumentar su miedo hacia el exterior, mientras que los de fuera pueden suponer una desconfianza discriminatoria de los que están adentro (CYTET, 2002, pág. 479).

No puede dejarse de lado el hecho de que la segregación residencial además de violar la libre circulación de la población, viola el derecho de los y las ciudadanas a gozar del espacio público de la ciudad.

De igual manera la segregación residencial actúa como un factor adicional de incremento de violencia: el mo- 
vimiento de los pobres por la ciudad los convierte en posibles delincuentes o, al menos, sospechosos, porque su extraterritorialidad los delata (Carrión, 2003, pág. 59).

Las urbanizaciones cerradas tienden a marcar segregación social y acentúan la condición de pobreza de aquellos que no tienen la posibilidad de acceder a ese tipo de urbanizaciones.

Uno de los elementos que debe tomarse en cuenta en este apartado es la escasa y hasta nula reglamentación dirigida a la construcción de este tipo de complejos residenciales. La Alcaldía Municipal del Distrito Central a través de la Gerencia de Control de la Construcción considera un reglamento que contempla las Normas Transitorias de actualización de la Zonificación y Normas de Fraccionamientos, Obras y Usos del Suelo en el Distrito Central que carece de la definición de fraccionamiento, barrio cerrado u urbanización cerrada, coyuntura que limita la compresión de la reglamentación en relación al tema.

\section{A manera de reflexión}

La fragmentación urbana es un fenómeno que incide no sólo en la morfología de la ciudad afectando su estructura y funcionamiento, también va un poco más allá puesto que de manera indirecta afecta las relaciones sociales que se establece entre los pobladores de una ciudad puesto que crea barreras tangibles e intangibles que fortalecen los problemas de exclusión social y territorial.

El Distrito Central en este proceso de fragmentación y segregación urbana ha comenzado a cerrarse y los espacios públicos urbanos a privatizarse y por ende a perderse, los muros, portones, plumas establecen límites y fronteras entre la población, generando un aislamiento que fortalece los sentimientos de individualismo, en gran parte de las ocasiones ni siquiera conocemos a nuestros vecinos, mucho menos tenemos la oportunidad de compartir parte de nuestro tiempo con ellos. Con la fragmentación del espacio se genera la fragmentación de la comunidad y se limita las oportunidades de diálogo y consenso entre la población.
El proceso de globalización ligado a la diversificación de las actividades comerciales y productivas ha fortalecido la fragmentación y segregación urbana en el municipio del Distrito Central, si bien es cierto la ciudad ha comenzado a desarrollar un estilo arquitectónico moderno también se denota las grandes diferencias entre los fragmentos urbanos por un lado los que carecen de los servicios e infraestructura básica y por el otro los que gozan de estos servicios que ofrece el Estado y en donde se observa la inversión en infraestructura urbana que elevan a la vez el valor de sus bienes, generando una fuerte diferenciación e inequidad social. Se hace necesario que el Estado y las autoridades competentes visualicen este proceso puesto que silenciosamente está separando a la población hondureña produciendo la disolución de la identidad de la ciudad en su conjunto.

La violencia e inseguridad indirectamente ha contribuido al proceso de fragmentación y segregación urbana, sin embargo, mas allá de toda la inversión que el Estado pueda hacer para combatirla es necesario revisar el problema desde sus orígenes estructurales pues solo en la medida que se identifiquen la raíz de este fenómeno podrá ser abordado de manera efectiva.

\section{Bibliografía}

- Álvarez, Horacio Gabriel (2005). Gran Buenos Aires Conurbado y Partido de San Martín: Exclusión Social y Segregación Urbana. Revista Electrónica Scripta Nova, volumen n⿳丷厂 IX n 194, Universidad de Barcelona.

- Bosford, A, Hidalgo R ( 2004) Formas Tempranas de Exclusión Residencial. El Modelo de Ciudad Cerrada en América Latina. El Caso de Santiago, en Revista de Geografía, Norte Grande, número 32 de la Pontificia Universidad Católica de Chile pag 21-37.

- Caballero Z, Elsa L (1984) El Metroplan y la Política de Vivienda 1976-1984.Universidad Nacional Autónoma de Honduras.

- Caldeira Teresa (1996). "Un nouveau modèle de ségrégation spatiale: les mursde Sao Paulo". En Revue Internationale des Sciences Sociales, núm.147, pp. 65-77. 
- Castells, Manuel (1999). La Cuestión Urbana, Editorial, siglo XXI, México.

- Carrión, Fernando (2003) Entre el Crimen y el Castigo. Seguridad Ciudadana y Control Democrático en América Latina y el Caribe. Revista Nueva Sociedad, Caracas, pp. 51-84.

- Ciccollella, Pablo (2002) La Metropolis Postsocial: Buenos Aires, Ciudad Rehén de la Economía Global. Actas del Seminario Internacional: El Desafío de las Áreas Metropolitanas en un Mundo Globalizado. Una Mirada a Europa y a América Latina, Barcelona, España.

- CYTET (2002) Ciudad y Territorios. Estudios Territoriales XXXIV. Urbanizaciones Encerradas, Pactos y Problemas. David Callies, Paula A. Franzese y Heidi Kai.

- De Mattos, C (2001). "Metropolización y suburbanización”. EURE (Santiago), (on line ), mayo, vol.27, no 80, pp 5-8, Chile.

- Estébanez, J. (1992) Los espacios urbanos. En Puyol, R et al: Geografía Humana. Ed Cátedra. Madrid, Espana.

- Giglia, Ángela (2002) Privatización del espacio, auto segregación y participación ciudadana en la ciudad de México: el caso de las calles cerradas en la zona de Coapa, México.

- Flores F, Manuel (2003) 50 Años de Crecimiento Demográfico Hondureño, Instituto de Investigaciones Económicas y Sociales, UNAH.

- Harvey, David (1997) Las ciudades Fragmentadas, Reportaje paginas 12; 23 de marzo 97, Buenos Aires, Argentina.

- INE (2001) Base de datos del XVI Censo de Población y V de Vivienda, Tegucigalpa, Honduras.

- INE (2002-2009) Encuesta Permanente de Hogares de Propósitos Múltiples, serie mayo 2002- 2009, Tegucigalpa, Honduras.

- Judd. D.R (1995): «The Rise of the New Walled Cities», in Helen Ligget \& David C. Perry (eds.,) Spatial Practices Critical Explorations in Social/ Spatial Theory Thousand Oaks: Sage.

- Kaztman, Rubén (2001). Exclusión Social y Segre- gación Residencial. Exposición Ciclo de Foros de Políticas Públicas, Montevideo Uruguay.

- Link, Felipe (2008). Fragmentación Urbana y Consecuencias Sociales en Revista Ciudades no 7 $^{\text {, pa- }}$ ginas 28-37, Red Nacional de Investigación Urbana, Puebla, México.

- McKenzie, R.D (1924). "The Ecological Approch to the Study of Human Community”; en The American Journal of Sociology, volumen 30, número 3, pp 287 301, The University of Chicago Press,USA

- Mediana C, Federico (1997) El centro comercial: Una burbuja de cristal. En Diálogos de la comunicación \#50 octubre, 1997. En http://www.felafaes.org/rev. dialogos/dialogos/50.htm,

- Petrot-Shapira, M (2000) "Segregación, Fragmentación Secesión. Hacia una Nueva Geografía Social en La Aglomeración de Buenos Aires". Revista Economía, Sociedad y Territorio, volumen II, número 7 pp 405-431.

- POSCAE-UNAH (2008) Centros Comerciales un Nuevo Punto de Encuentro: Privatización de los Espacios Publico. Elsa Lily Caballero Zeitún, Marysabel Zelaya.

- Rodríguez V, Jorge (2001). "Segregación residencial socioeconómica: ¿Qué es?, ¿Cómo se mide?, ¿Qué está pasando?, iImporta?", en Serie Población y Desarrollo no 16, , CEPAL/ECLAC Santiago de Chile.

- Sabatini, Francisco (2003) La Segregación Social del Espacio en las Ciudades de América Latina. BID "Desarrollo Social. Documento de Estrategia" Washington DC

- Valdés, Estela (2007) Fragmentación y Segregación Urbana: Aportes teóricos para el análisis de casos en la ciudad de Córdoba, Ponencia en Primer Congreso de Geografía de Universidades Nacionales. 05 al 08 de junio de 2007. Río Cuarto. Córdoba, Argentina.

- Vidal R, Rodrigo (1997) "Metrópolis en recomposición: Elementos para una teoría de la fragmentación urbana". En 6to Encuentro de Geógrafos de América Latina, Buenos Aires, marzo 1997. 